\title{
ON THE REPRESENTATION OF EFFECTIVE ENERGY DENSITIES
}

\author{
Christopher J. LARSEN ${ }^{1}$
}

\begin{abstract}
We consider the question raised in [1] of whether relaxed energy densities involving both bulk and surface energies can be written as a sum of two functions, one depending on the net gradient of admissible functions, and the other on net singular part. We show that, in general, they cannot. In particular, if the bulk density is quasiconvex but not convex, there exists a convex and homogeneous of degree 1 function of the jump such that there is no such representation.
\end{abstract}

AMS Subject Classification. 49Q15, 49Q20, 73M25, 73V25.

Received January 31, 2000. Revised August, 2000.

\section{INTRODUCTION}

This paper was motivated by a conjecture in [1] concerning the representation of effective energy densities. Though the statement of the conjecture is limited to densities corresponding to "structured deformations", it raises an interesting question about all densities that result from relaxation in $B V$, as we explain below. The question can be described as follows: suppose we have an energy

$$
E(u):=\int_{\Omega} W(\nabla u) d x+\int_{S_{u}} \phi([u], \nu) d \mathcal{H}^{N-1},
$$

where $\Omega \subset \mathbb{R}^{N}$ is Lipschitz, $u \in S B V\left(\Omega ; \mathbb{R}^{m}\right), \nabla u$ is the Radon-Nikodym derivative of $D u$ with respect to $\mathcal{L}^{N}$, $S_{u}$ is the complement of the set of Lebesgue points of $u,[u]$ is the jump in $u$ across $S_{u}, \nu$ is the normal to $S_{u}$, $\mathcal{H}^{N-1}$ is the $N-1$ dimensional Hausdorff measure, and $W, \phi$ are continuous with some growth assumptions.

We seek to minimize this energy (perhaps subject to some boundary conditions), and so we consider a minimizing sequence $\left\{u_{n}\right\}$, which will converge to some $u$. $u$ may not be a minimizer, but in a sense it does reflect energetic optimality, being the limit of an optimal sequence. We associate the limiting energy of the sequence $\left\{u_{n}\right\}$ with $u$, and call this the relaxed or effective energy $I(u)$. That is, $I(u)=\lim _{n \rightarrow \infty} E\left(u_{n}\right)$. I $(u)$ can be defined similarly even if $u$ is not the limit of a minimizing sequence, as follows:

$$
I(u):=\inf \left\{\liminf _{n \rightarrow \infty} E\left(u_{n}\right): u_{n} \rightarrow u\right\}
$$

Keywords and phrases: Relaxation, quasiconvexity, integral representation.

${ }^{1}$ Department of Mathematical Sciences, Worcester Polytechnic Institute, Worcester, MA 01609, U.S.A.;

e-mail: cjlarsen@wpi.edu 
A basic question in relaxation theory is to characterize $I(u)$, especially as an integral of new densities: an effective bulk density $W^{*}$, an effective interfacial density $\phi^{*}$, and perhaps others, so that, for example,

$$
I(u)=\int_{\Omega} W^{*}(\nabla u) d x+\int_{S_{u}} \phi^{*}([u], \nu) d \mathcal{H}^{N-1} .
$$

The model proposed in [4] concerns the fact that $\nabla u$ may differ from the appropriate limit of $\left\{\nabla u_{n}\right\}$. The reason for this is that the functions $u_{n}$ may have a considerable proportion of their variation on their jump sets $S_{u_{n}}$, so that $\nabla u_{n}$ does not reflect their variation, yet the limit $u$ may be smooth, or at least smooth in regions in which $u_{n}$ are not smooth. The point is that when the functions $u: \Omega \rightarrow \mathbb{R}^{m}$ represent deformations, the difference $\nabla u-\lim _{n \rightarrow \infty} \nabla u_{n}$ can be considered to be a measure of deformation due to "disarrangements". It is then natural to consider one energy density on these disarrangements and a separate density on smooth deformation, reflected in $\lim _{n \rightarrow \infty} \nabla u_{n}$. Motivated by this point of view [1] formulated the relaxation explicitly in terms of the limit of $\left\{\nabla u_{n}\right\}$. With the function $G$ representing a possible limit of $\left\{\nabla u_{n}\right\}$, they define

$$
I(u, G):=\inf \left\{\liminf _{n \rightarrow \infty} E\left(u_{n}\right):\left\{u_{n}\right\} \subset S B V\left(\Omega ; \mathbb{R}^{m}\right), u_{n} \rightarrow u \text { in } L^{1}\left(\Omega ; \mathbb{R}^{m}\right), \nabla u_{n} \stackrel{*}{\rightarrow} G \text { as measures }\right\},
$$

where we have ignored some technical requirements on the admissible sequences $\left\{u_{n}\right\}$. They show that the effective bulk term is

$$
\int_{\Omega} H(\nabla u(x), G(x)) d x
$$

and if $\phi$ is homogeneous of degree one, the effective bulk density $H$ is given by

$$
H(A, B):=\inf \left\{E(u): u \in S B V\left(Q ; \mathbb{R}^{m}\right),\left.u\right|_{\partial Q}=A x, \text { and } \int_{Q} \nabla u d x=B\right\},
$$

where $A, B \in \mathbb{M}^{m \times N}$, the space of $m \times N$ matrices, and $Q$ is a unit cube in $\mathbb{R}^{N}$.

The conjecture is that this density is a sum of a function of $G(x)$ (representing the limit of the elastic parts of the deformations $\left\{u_{n}\right\}$ ) and a function of $\nabla u(x)-G(x)$ (representing the limit of the disarrangement parts of the deformations). Precisely, $H(A, B)=F_{1}(B)+F_{2}(A-B)$ for some functions $F_{1}$ and $F_{2}$.

The consequences for general effective energies stem from the fact that typically, the effective bulk density $W^{*}$ is of the form

$$
W^{*}(A)=\inf \left\{E(u): u \in S B V\left(Q ; \mathbb{R}^{m}\right),\left.u\right|_{\partial Q}=A x\right\}
$$

which is evidently the same as

$$
\inf \left\{H(A, B): B \in \mathbb{M}^{m \times N}\right\}
$$

Since $H$ is generally continuous, any representation for $H$ would translate into a representation for $W^{*}$. A similar result holds for effective interfacial densities $\phi^{*}$.

We show that in general, there cannot be such a representation. The idea is the following. From (0.1), we see that $A$ reflects the net total derivative of the admissible functions, while $B$ gives the net gradient of admissible functions. $A-B$ is then the net singular part of admissible functions. The point is that the presence of some singular part of $D u$ can allow a decrease in $\int_{Q} W(\nabla u) d x$, even without a change in the net gradient. This is due to the fact that "gradients" of $B V$ functions need not be curl-free - they are not true gradients. $\int_{Q} W(\nabla u) d x$ can then approach the convex envelope of $W$ at $B$, whereas if there is no singular part, the $B V$ "gradient" is a true gradient, and $\int_{Q} W(\nabla u) d x$ cannot be less than $W(B)$ if $W$ is quasiconvex. But $W(B)$ may be strictly 
greater than the convex envelope of $W$ at $B$. Once it makes sense to have some singular part of $D u$, there is no reason to expect it to be energetically optimal to have zero net singular part, yet this is a consequence of the representation.

Consistent with the assumptions in [1], we assume that $W, \phi \geq 0, W(0)=\phi(0, \nu)=0$, and both functions are continuous. For simplicity, we take $N=m=2$, though the proof is straightforward to extend to higher dimensions. The first step is to prove

Lemma 0.1. If $H(A, B)=F_{1}(B)+F_{2}(A-B)$ for some functions $F_{1}, F_{2}$, then

$$
\min H(\cdot, B)=H(B, B)
$$

for all $B \in \mathbb{M}^{2 \times 2}$.

The contradiction to the representation then follows from

Theorem 0.2. If $W: \mathbb{M}^{2 \times 2} \rightarrow \mathbb{R}$ is quasiconvex but not convex and has growth $p \geq 1$, there exists $B \in \mathbb{M}^{2 \times 2}$ and a function $\phi: \mathbb{R}^{2} \times S^{1} \rightarrow \mathbb{R}$ that is linearly coercive, positive homogeneous of degree 1 , and subadditive in the first variable such that

$$
\min H(\cdot, B) \neq H(B, B) .
$$

These are proved in Section 3 below. Section 1 contains some preliminaries, and Section 2 develops some analysis on sequences in $B V$ that we use to prove Theorem 0.2.

\section{Preliminaries}

We consider a bounded, open set $\Omega \subset \mathbb{R}^{N}$ with Lipschitz boundary, and we define the spaces $L^{p}\left(\Omega ; \mathbb{R}^{m}\right)$, the Sobolev spaces $W^{1, p}\left(\Omega ; \mathbb{R}^{m}\right)$, and the space $B V\left(\Omega ; \mathbb{R}^{m}\right)$ in the usual way (see, e.g. [5]). For $u \in B V\left(\Omega ; \mathbb{R}^{m}\right)$, we define $|D u|(\Omega):=\sum_{i=1}^{m}\left|D u_{i}\right|(\Omega)$. From now on, we will usually just write $L^{p}(\Omega)$ for $L^{p}\left(\Omega ; \mathbb{R}^{m}\right)$, and similarly for $W^{1, p}$ and $B V$.

For $u \in B V(\Omega)$, we write $D u=D_{a c} u+D_{s} u$, where $D_{a c} u$ and $D_{s} u$ stand for, respectively, the absolutely continuous and singular part of $D u$ with respect to $\mathcal{L}^{N}$. We also consider the set $S_{u}$ of points which are not Lebesgue points for $u$, and recall that $S_{u}$ is $N$-1-rectifiable, and so it has a normal, $\nu, \mathcal{H}^{N-1}$-a.e. We use the representations $D_{a c} u=\nabla u \mathcal{L}^{N}$ and $\left(D_{s} u\right)\left\lfloor S_{u}=[u] \otimes \nu \mathcal{H}^{N-1}\left\lfloor S_{u}\right.\right.$, so we have the decomposition

$$
D u=\nabla u \mathcal{L}^{N}+[u] \otimes \nu \mathcal{H}^{N-1}\left\lfloor S_{u}+C(u),\right.
$$

where $C(u):=\left(D_{s} u\right)\left\lfloor\left(\Omega \backslash S_{u}\right),[u]\right.$ is the jump in $u$ across $S_{u}$, i.e. $[u]=u^{+}-u^{-}$, where $u^{+}$and $u^{-}$are the traces of $u$ on either side of $S_{u}$. If $C(u)=0$, then we say $u$ is a special function of bounded variation, and we write $u \in S B V(\Omega)$. This space was introduced in [3].

We denote the space of $m \times N$ matrices by $\mathbb{M}^{m \times N}$, and we consider $W: \mathbb{M}^{m \times N} \rightarrow \mathbb{R}$ and $\phi: \mathbb{R}^{m} \times S^{N-1} \rightarrow \mathbb{R}$. For $u \in B V\left(\Omega ; \mathbb{R}^{m}\right)$, we define

$$
E(u):=\int_{\Omega} W(\nabla u) d x+\int_{S_{u}} \phi([u], \nu) d \mathcal{H}^{N-1} .
$$

We say $W$ (and also $E$ ) has growth $p$ if there exist positive constants $c_{1}$ and $c_{2}$ such that

$$
c_{1}|A|^{p}-\frac{1}{c_{1}} \leq W(A) \leq c_{2}\left(|A|^{p}+1\right)
$$

for all $A \in \mathbb{M}^{m \times N}$. 
Recall that a function $W: \mathbb{M}^{m \times N} \rightarrow \mathbb{R}$ with growth $p$ is quasiconvex if

$$
W(A) \leq \int_{S} W(\nabla \varphi) d x
$$

for all $\varphi \in A x+W_{0}^{1, p}\left(S ; \mathbb{R}^{m}\right)$ and all $A \in \mathbb{M}^{m \times N}$, where $S \subset \mathbb{R}^{N}$ is open and satisfies $\mathcal{L}^{N}(S)=1$ (see [2]).

Throughout, we use $c$ to designate a constant that may change from line to line, depending only on $N, m$, and perhaps established uniform bounds. We will also use the fact that for $u, v \in B V(\Omega), \nabla u=\nabla v \mathcal{L}^{N}$ almost everywhere in the set $\{u=v\}$.

\section{SequenCES In BV}

We begin with some analysis of sequences in $B V$ that will be useful in proving Theorem 0.2 . The following is an extension of Lemma 1.2 in [6] to certain sequences in $B V$.

Lemma 2.1. Let $\left\{u_{i}\right\}$ be a bounded sequence in $B V(\Omega)$ such that $\left\{\nabla u_{i}\right\}$ is bounded in $L^{p}(\Omega)$ for some $p>1$ and $\left|D_{s} u_{i}\right|(\Omega) \rightarrow 0$. Then there exists a sequence $\left\{w_{i}\right\}$ bounded in $W^{1, p}(\Omega)$ such that $\left\{\left|\nabla w_{i}\right|^{p}\right\}$ is equiintegrable and

$$
\mathcal{L}^{N}\left(\left\{u_{i} \neq w_{i} \text { or } \nabla u_{i} \neq \nabla w_{i}\right\}\right) \rightarrow 0
$$

Proof. The proof is based on ideas from Theorem 2 in Section 6.6.2 and Theorem 3 in Section 6.6.3 of [5], together with Lemma 1.2 of [6]. We first define

$$
R_{i}^{\lambda}:=\left\{x \in \Omega: \frac{1}{\mathcal{L}^{N}(B(x, r))} \int_{B(x, r)} d\left|D u_{i}\right| \leq \lambda \quad \forall r>0\right\}
$$

and

$$
S_{i}^{\lambda}:=S_{i} \cup\left\{x \in \Omega:\left|\nabla u_{i}\right|(x)>\frac{\lambda}{2}\right\}
$$

where $S_{i}$ has measure zero and satisfies $\left|D_{s} u_{i}\right|\left(S_{i}\right)=\left|D_{s} u_{i}\right|(\Omega)$. For each $i \in \mathbb{N}$, we see from Vitali's covering theorem that there exists a countable set of disjoint balls $B\left(x_{j}, r_{j}\right)$ such that

$$
\Omega \backslash R_{i}^{\lambda} \subset \cup_{j=1}^{\infty} B\left(x_{j}, 5 r_{j}\right)
$$

and

$$
\frac{1}{\mathcal{L}^{N}\left(B\left(x_{j}, r_{j}\right)\right)} \int_{B\left(x_{j}, r_{j}\right)} d\left|D u_{i}\right|>\lambda
$$

We then have

$$
\lambda \mathcal{L}^{N}\left(\cup_{j=1}^{\infty} B\left(x_{j}, r_{j}\right)\right)<\left|D u_{i}\right|\left(\cup_{j=1}^{\infty} B\left(x_{j}, r_{j}\right)\right) .
$$

Now write

$$
\left|D u_{i}\right|\left(\cup_{j=1}^{\infty} B\left(x_{j}, r_{j}\right)\right)=\left|D u_{i}\right|\left(S_{i}^{\lambda} \cap \cup_{j=1}^{\infty} B\left(x_{j}, r_{j}\right)\right)+\left|D u_{i}\right|\left(\left[\Omega \backslash S_{i}^{\lambda}\right] \cap \cup_{j=1}^{\infty} B\left(x_{j}, r_{j}\right)\right),
$$


and it follows from the definition of $S_{i}^{\lambda}$ that

$$
\left|D u_{i}\right|\left(\left[\Omega \backslash S_{i}^{\lambda}\right] \cap \cup_{j=1}^{\infty} B\left(x_{j}, r_{j}\right)\right) \leq \frac{\lambda}{2} \mathcal{L}^{N}\left(\cup_{j=1}^{\infty} B\left(x_{j}, r_{j}\right)\right) .
$$

We therefore have, from (2.2),

$$
\lambda \leq \frac{1}{\mathcal{L}^{N}\left(\cup_{j=1}^{\infty} B\left(x_{j}, r_{j}\right)\right)}\left|D u_{i}\right|\left(S_{i}^{\lambda} \cap \cup_{j=1}^{\infty} B\left(x_{j}, r_{j}\right)\right)+\frac{\lambda}{2}
$$

and so

$$
\lambda \leq \frac{2}{\mathcal{L}^{N}\left(\cup_{j=1}^{\infty} B\left(x_{j}, r_{j}\right)\right)}\left|D u_{i}\right|\left(S_{i}^{\lambda} \cap \cup_{j=1}^{\infty} B\left(x_{j}, r_{j}\right)\right)
$$

and

$$
\mathcal{L}^{N}\left(\cup_{j=1}^{\infty} B\left(x_{j}, r_{j}\right)\right) \leq \frac{2}{\lambda}\left|D u_{i}\right|\left(S_{i}^{\lambda} \cap \cup_{j=1}^{\infty} B\left(x_{j}, r_{j}\right)\right) .
$$

We now have that

$$
\begin{aligned}
\mathcal{L}^{N}\left(\Omega \backslash R_{i}^{\lambda}\right) & \leq c \mathcal{L}^{N}\left(\cup_{j=1}^{\infty} B\left(x_{j}, r_{j}\right)\right) \leq \frac{c}{\lambda}\left|D u_{i}\right|\left(S_{i}^{\lambda} \cap \cup_{j=1}^{\infty} B\left(x_{j}, r_{j}\right)\right) \leq \frac{c}{\lambda}\left[\left|D_{s} u_{i}\right|(\Omega)+\int_{S_{i}^{\lambda}}\left|\nabla u_{i}\right| d x\right] \\
& \leq \frac{c}{\lambda}\left[\left|D_{s} u_{i}\right|(\Omega)+\left(\int_{S_{i}^{\lambda}}\left|\nabla u_{i}\right|^{p} d x\right)^{\frac{1}{p}} \mathcal{L}^{N}\left(S_{i}^{\lambda}\right)^{1-\frac{1}{p}}\right] .
\end{aligned}
$$

We are given that $\int_{S_{i}^{\lambda}}\left|\nabla u_{i}\right|^{p} d x \leq M$ for some $M>0$, and from the definition of $S_{i}^{\lambda}$ we have that

$$
\int_{S_{i}^{\lambda}}\left|\nabla u_{i}\right|^{p} d x \geq \mathcal{L}^{N}\left(S_{i}^{\lambda}\right)\left(\frac{\lambda}{2}\right)^{p}
$$

so that

$$
\mathcal{L}^{N}\left(S_{i}^{\lambda}\right) \leq \frac{c}{\lambda^{p}} .
$$

We now have

$$
\mathcal{L}^{N}\left(\Omega \backslash R_{i}^{\lambda}\right) \leq \frac{c}{\lambda}\left[\left|D_{s} u_{i}\right|(\Omega)+\left(\int_{S_{i}^{\lambda}}\left|\nabla u_{i}\right|^{p} d x\right)^{\frac{1}{p}} \frac{1}{\lambda^{p-1}}\right] \leq \frac{c}{\lambda}\left|D_{s} u_{i}\right|(\Omega)+\frac{c}{\lambda^{p}} .
$$

Hence,

$$
\lambda^{p} \mathcal{L}^{N}\left(\Omega \backslash R_{i}^{\lambda}\right) \leq c \lambda^{p-1}\left|D_{s} u_{i}\right|(\Omega)+c .
$$

We then choose $\lambda(i)$ such that

$$
\lambda(i)^{p-1}\left|D_{s} u_{i}\right|(\Omega)=1
$$


and $\lambda(i)^{p} \mathcal{L}^{N}\left(\Omega \backslash R_{i}^{\lambda(i)}\right)$ is then bounded. From the proof of Theorem 2 in Section 6.6.2 of [5], we know that there exist Lipschitz functions $v_{i}$ with $\operatorname{Lip}\left(v_{i}\right) \leq c \lambda(i)$ and $v_{i}=u_{i}$ on $R_{i}^{\lambda(i)}$. Hence,

$$
\int_{\Omega}\left|\nabla v_{i}\right|^{p} d x \leq \int_{R_{i}^{\lambda(i)}}\left|\nabla u_{i}\right|^{p} d x+c \lambda(i)^{p} \mathcal{L}^{N}\left(\Omega \backslash R_{i}^{\lambda(i)}\right)
$$

and $\left\{\nabla v_{i}\right\}$ is bounded in $L^{p}(\Omega)$. Note that since $\left\{u_{i} \neq v_{i}\right\} \subset \Omega \backslash R_{i}^{\lambda(i)}$, we have

$$
\mathcal{L}^{N}\left(\left\{u_{i} \neq v_{i}\right\}\right) \rightarrow 0
$$

It now follows from the fact that $\left\{u_{i}\right\}$ is bounded in $L^{1}(\Omega)$ and the Poincare inequality that $\left\{v_{i}\right\}$ is bounded in $W^{1, p}(\Omega)$. To see this, suppose $\left\|v_{i}\right\|_{p} \rightarrow \infty$. Set $y_{i}:=\frac{v_{i}}{\left\|v_{i}\right\|_{p}}$, so $\left\|y_{i}\right\|_{p}=1$ and $\left\|\nabla y_{i}\right\|_{p} \rightarrow 0$. Hence, $y_{i} \rightarrow y_{\infty}$ in $L^{p}$, and $y_{\infty}$ is a constant. Now set $z_{i}:=\frac{u_{i}}{\left\|v_{i}\right\|_{p}}$. Then $\mathcal{L}^{N}\left(\left\{z_{i} \neq y_{i}\right\}\right) \rightarrow 0$ and $\left\|z_{i}\right\|_{1} \rightarrow 0$. Therefore, $y_{\infty}=0$, contradicting $\left\|y_{i}\right\|_{p}=1$.

By Lemma 1.2 in [6], there is a sequence $\left\{w_{i}\right\}$ bounded in $W^{1, p}(\Omega)$ with

$$
\mathcal{L}^{N}\left(\left\{v_{i} \neq w_{i}\right\}\right) \rightarrow 0
$$

and $\left\{\left|\nabla w_{i}\right|^{p}\right\}$ is equiintegrable. We then also have $\mathcal{L}^{N}\left(\left\{u_{i} \neq w_{i}\right\}\right) \rightarrow 0$, and $\left\{w_{i}\right\}$ satisfies the conclusion of the lemma.

The following is an easy consequence. Note that the conclusion below is false when $p=1$, the idea being that concentrations in $\left\{\left|\nabla u_{i}\right|\right\}$ can serve a purpose - they can cause nonsmooth variations in the limit, whereas effects of concentrations only in $\left\{\left|\nabla u_{i}\right|^{p}\right\}$ disappear in the limit.

Remark 2.2. If $\left\{u_{i}\right\}$ is a sequence in $B V(\Omega)$ with $\left|D_{s} u_{i}\right|(\Omega) \rightarrow 0$ and minimizing an energy with growth $p>1$, then $\left\{\left|\nabla u_{i}\right|^{p}\right\}$ is equiintegrable.

We first note that a sequence $\left\{u_{i}\right\}$ in $W^{1, p}(\Omega)$ minimizing an energy with growth $p>1$ satisfies the hypotheses of the remark, but equiintegrability for that case could be proved directly from Lemma 1.2 in [6], with no need for Lemma 2.1 here.

To see the conclusion of the remark, suppose we have a sequence $\left\{u_{i}\right\}$ such that $\left\{\left|\nabla u_{i}\right|^{p}\right\}$ is not equiintegrable. Then there exists $\delta>0$ and a sequence of sets $\left\{E_{i}\right\}$ such that $\int_{E_{i}}\left|\nabla u_{i}\right|^{p} d x \geq \delta$ and $\mathcal{L}^{N}\left(E_{i}\right) \rightarrow 0$. We then use Lemma 2.1 to find $\left\{w_{i}\right\}$ equiintegrable and such that, for $T_{i}:=\left\{u_{i} \neq w_{i}\right\}$, we have $\mathcal{L}^{N}\left(T_{i}\right) \rightarrow 0$. Then

$$
\begin{aligned}
\int_{\Omega} W\left(\nabla u_{i}\right) d x & =\int_{\Omega \backslash\left(E_{i} \cup T_{i}\right)} W\left(\nabla w_{i}\right) d x+\int_{E_{i} \cup T_{i}} W\left(\nabla u_{i}\right) d x \\
& =\int_{\Omega} W\left(\nabla w_{i}\right) d x-\int_{E_{i} \cup T_{i}} W\left(\nabla w_{i}\right) d x+\int_{E_{i} \cup T_{i}} W\left(\nabla u_{i}\right) d x \\
& \geq \int_{\Omega} W\left(\nabla w_{i}\right) d x-c_{2} \int_{E_{i} \cup T_{i}}\left|\nabla w_{i}\right|^{p} d x-c_{2} \mathcal{L}^{N}\left(E_{i} \cup T_{i}\right)+c_{1} \delta-\frac{1}{c_{1}} \mathcal{L}^{N}\left(E_{i} \cup T_{i}\right)
\end{aligned}
$$

so that, since $\left\{\left|\nabla w_{i}\right|^{p}\right\}$ is equiintegrable,

$$
\liminf _{i \rightarrow \infty} \int_{\Omega} W\left(\nabla u_{i}\right) d x \geq \liminf _{i \rightarrow \infty} \int_{\Omega} W\left(\nabla w_{i}\right) d x+c_{1} \delta
$$

and $\left\{u_{i}\right\}$ is not minimizing.

We now have the following, which is key to proving Theorem 0.2. 
Lemma 2.3. Let $\beta>0, W$ quasiconvex with growth $p \geq 1$, and $A \in \mathbb{M}^{m \times N}$ be given. Then there exists $C>0$ such that if $u \in B V(Q)$ and

$$
\begin{aligned}
& \text { i) }\left.u\right|_{\partial Q}=A x \\
& \text { ii) } \quad \int_{Q} W(\nabla u) d x \leq W(A)-\beta
\end{aligned}
$$

then $\left|D_{s} u\right|(Q)>C$.

Proof. We suppose to the contrary that for $i \in \mathbb{N}$ there exists $u_{i} \in B V(Q)$ satisfying i) and ii), but $\left|D_{s} u_{i}\right|(Q)<$ $\frac{1}{i}$. If $p>1$, then $\left\{u_{i}\right\}$ satisfies the hypotheses of Lemma 2.1, and so we choose a sequence $\left\{w_{i}\right\}$ as allowed by that lemma. Since $\left\{w_{i}\right\}$ is bounded in $W^{1, p}(\Omega)$, there is a subsequence such that $w_{i} \rightarrow w$ in $W^{1, p}(\Omega)$ for some $w$. Since a subsequence of $\left\{u_{i}\right\}$ converges in $L^{1}(\Omega)$ to the same function with $\left|D_{s} u_{i}\right|(Q) \rightarrow 0$ and $\left\{\nabla u_{i}\right\}$ bounded in $L^{p}(\Omega)$, we have

$$
\left.w\right|_{\partial Q}=A x
$$

Yet, since $\mathcal{L}^{N}\left(\left\{u_{i} \neq w_{i}\right\}\right) \rightarrow 0$ and $\left\{\left|\nabla w_{i}\right|^{p}\right\}$ is equiintegrable, we have

$$
\liminf _{i \rightarrow \infty} \int_{Q} W\left(\nabla w_{i}\right) d x \leq \liminf _{i \rightarrow \infty} \int_{Q} W\left(\nabla u_{i}\right) d x \leq W(A)-\beta .
$$

From the weak lower semicontinuity of integrals of quasiconvex functions, we then get

$$
W(A) \leq \int_{Q} W(\nabla w) d x \leq \liminf _{i \rightarrow \infty} \int_{Q} W\left(\nabla w_{i}\right) d x \leq W(A)-\beta,
$$

a contradiction. We note that, as pointed out by the referee, this case also follows from results in [7], without the need for Lemma 2.1 .

For the case $p=1$, we get the same contradiction using Lemma 3.1 in [8] and rescaling $\left\{u_{i}\right\}$ so that the rescaled sequence converges to $A x$ in $L^{1}(\Omega)$ without changing $\int_{Q} W\left(\nabla u_{i}\right) d x$.

\section{THE REPRESENTATION THEOREM}

We now use the previous analysis of $B V$ sequences to prove Theorem 0.2. The contradiction to the representation will come from the following.

Lemma 0.1 If $H(A, B)=F_{1}(B)+F_{2}(A-B)$ for some functions $F_{1}, F_{2}$, then

$$
\min H(\cdot, B)=H(B, B)
$$

for all $B \in \mathbb{M}^{2 \times 2}$.

Proof. Suppose there are such $F_{1}$ and $F_{2}$. It is immediate from the definition $(0.1)$ and the fact that $W, \phi \geq 0$ with $W(0)=\phi(0, \nu)=0$ that $H \geq 0$ and $H(0,0)=0$. It follows that $F_{1}(0)+F_{2}(0)=0$, or $F_{1}(0)=-F_{2}(0)$. We can then assume that

$$
F_{1}(0)=F_{2}(0)=0
$$


since by considering

$$
F_{1}^{\prime}(A):=F_{1}(A)-F_{1}(0)
$$

and corresponding $F_{2}^{\prime}$, we have the representation

$$
H(A, B)=F_{1}^{\prime}(B)+F_{2}^{\prime}(A-B)
$$

with $F_{1}^{\prime}(0)=F_{2}^{\prime}(0)=0$.

Now, since

$$
\begin{gathered}
H \geq 0, \\
H(B, B)=F_{1}(B)+F_{2}(0)=F_{1}(B),
\end{gathered}
$$

and

$$
H(A, 0)=F_{1}(0)+F_{2}(A)=F_{2}(A),
$$

it follows that $F_{1} \geq 0$ and $F_{2} \geq 0$. Therefore,

$$
H(A, B)=F_{1}(B)+F_{2}(A-B) \geq F_{1}(B)=H(B, B)
$$

which gives (3.3).

Below we show that (3.3) is not, in general, true. We first note that from [9], we know that there exists a quasiconvex function $W$ that is homogeneous of degree 1 but not convex, and so $W$ satisfies the hypotheses of Theorem 0.2 .

Theorem 0.2. If $W: \mathbb{M}^{2 \times 2} \rightarrow \mathbb{R}$ is quasiconvex but not convex and has growth $p \geq 1$, then there exists $B \in \mathbb{M}^{2 \times 2}$ and a continuous function $\phi: \mathbb{R}^{2} \times S^{1} \rightarrow \mathbb{R}$ that is linearly coercive, positive homogeneous of degree 1 , and subadditive in the first variable such that

$$
\min H(\cdot, B) \neq H(B, B) .
$$

Proof. For $\varepsilon, K>0$ to be chosen later, set

$$
\phi(\xi, \nu):=\sum_{j=1}^{2}\left[\varepsilon \sum_{k=1}^{2}\left(\xi_{k} \nu \cdot e_{j}\right)^{+}+K \sum_{k=1}^{2}\left(\xi_{k} \nu \cdot e_{j}\right)^{-}\right],
$$

which is linearly coercive, homogeneous of degree one, and subadditive in $\xi$. We then choose $B \in \mathbb{M}^{2 \times 2}$ such that $C W(B)<W(B)$, where $C W$ is the convex hull of $W$. Then we can write $B$ as a convex combination of $B_{i}$ 's, $B=\sum \lambda_{i} B_{i}$, with $\lambda_{i}$ rational and such that $\sum \lambda_{i} W\left(B_{i}\right)<W(B)$. We will now use this $B$ to construct matrices $A$ and $B^{\prime}$ and a function $v^{\prime}$ admissible for $H\left(A, B^{\prime}\right)$ with energy less than $H\left(B^{\prime}, B^{\prime}\right)$.

Set $\gamma_{k}:=\max _{i}\left\|B_{i}^{k} x\right\|_{L^{\infty}(Q)}$, where $B_{i}^{k}$ is the $k$ th row of $B_{i}, k=1,2$, and $Q$ is the cube $(0,1)^{2}$. We then set $\gamma:=\max _{k} \gamma_{k}$. Put an $n \times n$ grid on $Q$, where $n$ is a multiple of all the denominators of the $\lambda_{i}$ and will be chosen later. We now define $v \in S B V\left(Q ; \mathbb{R}^{2}\right)$. For $l=1, \ldots, n$, define $v$ on $\left(\frac{l-1}{n}, \frac{l}{n}\right) \times\left(0, \frac{1}{n}\right)$ by $\nabla v=B_{i_{l}}$ and, 
writing $v$ in terms of its components, $v=\left(v_{1}, v_{2}\right)$, we have $\min v_{1}=\min v_{2}=\frac{(l-1)}{n} \gamma$. Here the $B_{i_{l}}$ are chosen so that for $l=1, \ldots, \lambda_{1} n$ we have $B_{i_{l}}=B_{1}$, and so on, so that each $B_{i}$ is the gradient of $v$ in $\lambda_{i} n$ of the $n$ squares $\left(\frac{l-1}{n}, \frac{l}{n}\right) \times\left(0, \frac{1}{n}\right), l=1, \ldots, n$. We then extend $v$ to $(0,1) \times\left(\frac{1}{n}, 1\right)$ by $v_{k}(x):=v_{k}\left(x-\frac{l-1}{n} e_{2}\right)+\frac{l-1}{n} \gamma$, $k=1,2$ for $x \in(0,1) \times\left(\frac{l-1}{n}, \frac{l}{n}\right), l=2, \ldots, n$.

From the definition of $\gamma$ and the construction of $v$, it follows that $\left[v_{k}\right] \nu \cdot e_{j} \geq 0$ for $j, k=1,2$. Notice that, setting $A$ to be the $2 \times 2$ matrix with every entry $\gamma$, we have $\|v-A x\|_{L^{\infty}(\partial Q)} \leq 2 \frac{\gamma}{n}$. It also follows that $\left|D_{s} v\right|(Q)<8 \gamma$. To see this, notice that the variation of each component $v_{k}$ (by which we mean $\sup v_{k}-\inf v_{k}$ ) in each square of the grid is at most $\frac{\gamma}{n}$, and by construction of $v$, the variation over two adjacent squares is at most $2 \frac{\gamma}{n}$. Therefore, $\left|\left[v_{k}\right]\right| \leq 2 \frac{\gamma}{n}$ between adjacent squares. Furthermore, since

$$
S_{v_{k}} \subset[\{1 / n, 2 / n, \ldots,(n-1) / n\} \times(0,1)] \cup[(0,1) \times\{1 / n, 2 / n, \ldots,(n-1) / n\}],
$$

we have $\mathcal{H}^{1}\left(S_{v_{k}}\right) \leq 2(n-1)$. Hence, $\left|D_{s} v_{k}\right|(Q) \leq 2 \frac{\gamma}{n} 2(n-1)<4 \gamma$ and $\left|D_{s} v\right|(Q)<8 \gamma$.

We extend $v$ outside $Q$ by $v(x)=A x$, and for $\delta>0$, define $v_{\delta}(x):=v((1+\delta)\{x-\bar{x}\}+\bar{x})$, where $\bar{x}$ is the center of $Q$. Then $W\left(\int_{Q} \nabla v_{\delta} d x\right) \rightarrow W(B)$ and $\int_{Q} W\left(\nabla v_{\delta}\right) d x \rightarrow \sum_{i} \lambda_{i} W\left(B_{i}\right)$ as $\delta \rightarrow 0$. We choose $\delta$ so that $W\left(\int_{Q} \nabla v_{\delta} d x\right)>\int_{Q} W\left(\nabla v_{\delta}\right) d x$ and rename this $v_{\delta} v^{\prime}$.

Set $\alpha:=W\left(\int_{Q} \nabla v^{\prime} d x\right)-\int_{Q} W\left(\nabla v^{\prime}\right) d x$ and $B^{\prime}:=\int_{Q} \nabla v^{\prime} d x$. We now have that $v^{\prime}$ is admissible for $H\left(A, B^{\prime}\right)$ and

$$
\int_{S_{v^{\prime}}} \phi\left(\left[v^{\prime}\right], \nu\right) d \mathcal{H}^{1}<2 \varepsilon\left|D_{s} v\right|(Q)+8 K \frac{\gamma}{n},
$$

where the last term comes from the fact that $\|v-A x\|_{L^{\infty}(\partial Q)} \leq 2 \frac{\gamma}{n}$.

We choose $\varepsilon>0$ such that

$$
2 \varepsilon\left|D_{s} v\right|(Q)<\frac{\alpha}{3}
$$

Now, any $u$ admissible for $H\left(B^{\prime}, B^{\prime}\right)$ requires zero net jump, and so the jumps will be just as much in the minus $e_{j}$ directions as in the positive. That is,

$$
\int_{Q} d D_{s} u=0
$$

implies

$$
\int_{Q}\left[u_{k}\right] \nu \cdot e_{j} d \mathcal{H}^{1}=0
$$

so that

$$
\int_{Q}\left(\left[u_{k}\right] \nu \cdot e_{j}\right)^{+} d \mathcal{H}^{1}=\int_{Q}\left(\left[u_{k}\right] \nu \cdot e_{j}\right)^{-} d \mathcal{H}^{1}
$$

for $j, k \in\{1,2\}$. Hence

$$
E(u)>\frac{1}{2} K\left|D_{s} u\right|(Q) .
$$

From Lemma 2.3 with $\beta=\frac{\alpha}{3}$, there exists $C>0$ such that if $u$ is admissible for $H\left(B^{\prime}, B^{\prime}\right)$ and satisfies

$$
\int_{Q} W(\nabla u) d x \leq W\left(B^{\prime}\right)-\frac{\alpha}{3}
$$


then $\left|D_{s} u\right|(Q) \geq C$. An easy calculation shows that if $K>\frac{2}{C}\left(W\left(B^{\prime}\right)-\frac{\alpha}{3}\right)$, then $u \in S B V\left(Q ; \mathbb{R}^{2}\right)$ with trace $B^{\prime} x$ and $\int_{Q} \nabla u d x=B^{\prime}$ implies

$$
E(u)>W\left(B^{\prime}\right)-\frac{\alpha}{3}
$$

Choosing $n$ large enough so that $8 K \frac{\gamma}{n}<\frac{\alpha}{3}$ gives $H\left(A, B^{\prime}\right)<W\left(B^{\prime}\right)-\frac{\alpha}{3}$ with $H\left(B^{\prime}, B^{\prime}\right) \geq W\left(B^{\prime}\right)-\frac{\alpha}{3}$, contradicting $\min H\left(\cdot, B^{\prime}\right)=H\left(B^{\prime}, B^{\prime}\right)$ from Lemma 0.1.

\section{REFERENCES}

[1] R. Choksi and I. Fonseca, Bulk and interfacial energy densities for structured deformations of continua. Arch. Rational Mech. Anal. 138 (1997) 37-103.

[2] B. Dacorogna, Direct Methods in the Calculus of Variations. Springer-Verlag, Berlin (1989).

[3] E. De Giorgi and L. Ambrosio, Un nuovo tipo di funzionale del calcolo delle variazioni. Atti. Accad. Naz. Lincei Cl. Sci. Fis. Mat. Natur. Rend. Lincei (9) Suppl. 82 (1988) 199-210.

[4] G. Del Piero and D.R. Owen, Structured deformations of continua. Arch. Rational Mech. Anal. 124 (1993) 99-155.

[5] L.C. Evans and R.F. Gariepy, Measure Theory and Fine Properties of Functions. CRC Press, Boca Raton (1992).

[6] I. Fonseca, S. Müller and P. Pedregal, Analysis of concentration and oscillation effects generated by gradients. SIAM J. Math. Anal. 29 (1998) 736-756.

[7] J. Kristensen, Lower semicontinuity in spaces of weakly differentiable functions. Math. Ann. 313 (1999) 653-710.

[8] C.J. Larsen, Quasiconvexification in $W^{1,1}$ and optimal jump microstructure in BV relaxation. SIAM J. Math. Anal. 29 (1998) 823-848.

[9] S. Müller, On quasiconvex functions which are homogeneous of degree 1. Indiana Univ. Math. J. 41 (1992) $295-301$. 